\title{
MEDICIONES ALTERNATIVAS DE POBREZA EN CHILE, 1990-2015
}

\author{
ALTERNATIVE POVERTY MEASURES IN CHILE, 1990-2015
}

MEDIÇÕES ALTERNATIVAS DE POBREZA NO CHILE, 1990-2015

\author{
Gonzalo D. Martner ${ }^{1}$
}

\section{Resumen}

Chile ha disminuido desde la década de 1990 sus niveles de pobreza, en un contexto de crecimiento sostenido del PIB y de los ingresos de las familias. La intensidad de esa caída es materia de controversia, según las diversas metodologías de medición utilizadas. Se compara los principales índices de pobreza absoluta, relativa y multidimensional y se concluye que los resultados de las mediciones, a partir de una misma encuesta de ingresos, son altamente dependientes de las definiciones de pobreza. Se presenta evidencia para el período 1990-2015.

Palabras clave: ingresos; pobreza absoluta; pobreza relativa; pobreza multidimensional.

\begin{abstract}
Poverty levels in Chile have decreased since 1990, in a context of sustained GDP and family income growth. The intensity of this evolution is controversial based on the different measure methods applied. Absolute, relative, and multidimensional poverty indexes are compared. It is concluded that, based on the same income survey, resulting measures highly depend on poverty definitions. Evidence is presented for the 19902015 period.
\end{abstract}

Doi: http://dx.doi.org/10.15359/eys.23-53.7

Fecha de recepción: 09-01-2018. Fechas de reenvíos: 11-01-2018, 15-03-2018, 11-05-2018. Aceptado el 01-06-2018. Publicado el 01-06-2018.

${ }^{1}$ Economista. Director del Centro de Políticas para el Desarrollo y académico, Universidad de Santiago de Chile, Chile. Correo electrónico: gonzalo.martner@usach.cl

Gonzalo D. Martner

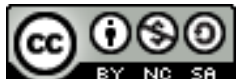

Revista Economía y Sociedad by Universidad Nacional is licensed under a CreativeCommons Reconocimiento-NoComercial- 
Keywords: income; absolute poverty; relative poverty; multidimensional poverty.

\section{Resumo}

Desde a década de 1990, o Chile reduziu seus níveis de pobreza, em um contexto de crescimento sustentado do PIB e da renda familiar. A intensidade desta queda é controversa de acordo com as diferentes metodologias de medição usadas. Comparam-se os principais índices de pobreza absoluta, relativa e multidimensional e conclui-se que os resultados das medições, baseados na mesma pesquisa de renda, são altamente dependentes das definições de pobreza. Evidências são apresentadas para o período 1990-2015.

Palavras-chave: renda; pobreza absoluta; pobreza relativa; pobreza multidimensional.

\section{Introducción}

Sen (1992) sostiene que "no hay razón alguna para suponer que la idea de pobreza deba ser tajante y precisa" (p. 2) y que "es posible que haya que usar más de un criterio en vista de la falta de uniformidad en los estándares aceptados" (p. 4). En la actualidad, diversos organismos internacionales -como el Banco Mundial, la Comisión Económica para América Latina y el Caribe de las Naciones Unidas (CEPAL) y la Organización para la Cooperación y el Desarrollo (OCDE)- y muchos servicios estadísticos nacionales utilizan criterios disímiles de medición de la incidencia de la pobreza en la población. En este texto se muestra, en el caso de Chile, las amplias diferencias en el resultado de estas mediciones según la definición de pobreza que se adopte.

\section{Metodologías de medición de la pobreza por el método del ingreso}

La metodología utilizada consiste en dimensionar esas diferencias contrastando las definiciones de la línea de pobreza de los tres organismos internacionales mencionados y la de los gobiernos chilenos entre 1990 y 2015. Todas estas entidades utilizan para medir la incidencia de la pobreza el método del ingreso a partir de la misma base de microdatos sobre los ingresos de las familias; es decir, la encuesta periódica de Caracterización Socioeconómica (CASEN) que realiza el gobierno de Chile.

La tasa de incidencia de la pobreza de ingresos (TI) es definida como la proporción de la población a la que se identifica como pobre porque sus ingresos son inferiores a una línea de pobreza especificada. Si $q$ es el número de personas identificadas como pobres y $n$ el número total de personas en la comunidad, entonces $T l=q / n$. Este índice es el más utilizado, a pesar de que, como observa Sen (1992), este índice t ignora la magnitud de los déficits de ingreso respecto 128 
a la línea de pobreza, o intensidad de la pobreza, la que se puede medir como "brecha de pobreza"; es decir, el déficit porcentual del ingreso medio de los pobres con respecto a la línea de pobreza.

La variable que se utiliza de modo más frecuente para los análisis de pobreza es el ingreso anual autodeclarado por las familias, sin considerar sus activos. Las personas que son encuestadas mediante métodos aleatorios para conformar muestras representativas entregan una información sobre sus ingresos y los de su familia de acuerdo con lo que conocen o estiman en la materia. La información con que cuenta el grupo familiar respecto de sus ingresos, que normalmente es homogénea cuando las personas que perciben ingresos desempeñan un trabajo dependiente y formal, o bien, reciben una pensión, es más heterogénea cuando los ingresos corresponden a rentas del capital, que normalmente no se liquidan mensualmente o son parcialmente registradas o a trabajos no dependientes o informales, cuyas rentas son variables y también carecen de registro $y$, por tanto, de capacidad de retención en base anual para el entrevistado seleccionado muestralmente. Las personas de mayores ingresos son menos proclives a responder las encuestas, lo que genera otro sesgo.

La metodología más antigua es la que busca medir la pobreza absoluta. El indicador convencional de pobreza utilizado oficialmente en Estados Unidos, por ejemplo, está definido por el contraste entre el valor monetario de una canasta de alimentos, cuya composición nutricional permite que un adulto mantenga su condición vital al consumirla y sus ingresos efectivos, multiplicado por un determinado factor que registre otras necesidades. Este es de tres veces en Estados Unidos y marca la línea de pobreza, proporción basada en el peso de los alimentos en el consumo total en un año base. La línea de pobreza de Estados Unidos fue creada en 1963-64 a partir de la observación de que el costo de la alimentación representaba por entonces un tercio de los ingresos familiares, valor que se indexa anualmente por la inflación, sin considerar las variaciones en el tiempo y en los distintos territorios del valor de los consumos distintos de la alimentación como la vivienda, el transporte y los servicios básicos. Por su parte, autores como Meyer \& Sullivan (2012) y Arrow (2015) abogan por utilizar en Estados Unidos las encuestas de consumo de los hogares para apreciar directamente el acceso a bienes y servicios antes que indirectamente a través de los ingresos, mientras Sen (1992) observa que las necesidades de alimentación varían de acuerdo con la condición física, las condiciones climáticas y los hábitos de trabajo en diversas sociedades, por lo que la idea de requerimientos nutricionales mínimos encierra "una arbitrariedad intrínseca", a la vez que "resulta difícil definir los requerimientos mínimos para los rubros no alimentarios".

Las estimaciones de mayor amplitud con este método del ingreso son las realizadas por el Banco Mundial (Ravallion \& Chen, 2011; Ravallion, 2011), organismo que define la pobreza como la proporción de la población cuyos ingresos son inferiores a la llamada "línea internacional de pobreza" en base al valor promedio de bienes de consumo indispensable en los quince países 
más pobres (actualizada en 2015 a 1,9 dólares por habitante diario a paridad de poder adquisitivo de 2011). A esta línea de pobreza (que también se basa en encuestas de ingresos de los hogares, o bien, de consumo cuando estas no existen, pues estas últimas están disponibles en casi todos los países para hacer la medición periódica de la evolución de los precios al consumidor) se agregaron, desde octubre de 2017, dos líneas adicionales de 3,2 y 5,5 dólares por habitante al día, las que reflejan la capacidad de compra promedio de bienes básicos en los países de ingresos bajos y medios, respectivamente.

La OCDE realiza mediciones de pobreza relativa con base en la metodología de la Unión Europea y considera umbrales como porcentaje de la mediana de ingresos familiares, estimando el porcentaje de la población en situación de pobreza considerando los hogares con ingresos inferiores al $50 \%$ y $60 \%$ de la mediana del ingreso disponible, tanto antes como después del pago de impuestos y la realización de transferencias monetarias a los hogares. Una tasa de pobreza relativa es un indicador de desigualdad de ingresos concentrado en la parte inferior de la distribución: mientras más débil es el nivel de vida de los más desfavorecidos en relación con el del resto de la población, la tasa de pobreza relativa será más elevada. Un aumento homogéneo de los ingresos permite un retroceso de la pobreza absoluta, pero mantiene una estabilidad de la pobreza relativa, lo demás permaneciendo constante.

Más recientemente, se ha agregado la metodología de pobreza multidimensional propuesta por Alkire (2007) \& Foster (2011), adaptada por países como México, Colombia y Chile y, más ampliamente, por el Índice de Pobreza Multidimensional del Programa de Naciones Unidas para el Desarrollo. Este índice identifica carencias a nivel de los hogares y las personas en los ámbitos de la salud, la educación y el nivel de vida. Utiliza microdatos de encuestas de la situación social de los hogares y cada miembro de una familia es clasificado como pobre o no pobre, en función del número de carencias que experimente su hogar. Estos datos se agregan para conformar el indicador de la pobreza multidimensional. El índice refleja tanto la prevalencia de las carencias multidimensionales como su intensidad; es decir, cuántas carencias sufren las personas al mismo tiempo, y ofrece un complemento a las herramientas de medición de la pobreza basadas en los ingresos.

\section{La disminución de la pobreza de ingresos en Chile}

En Chile, los organismos oficiales han considerado, desde la década de 1980 hasta 2013, que un hogar está en situación de pobreza extrema si su ingreso mensual por persona es inferior al ingreso mínimo establecido para satisfacer las necesidades alimentarias de una persona. A su vez, un hogar se ha considerado en situación de pobreza si su ingreso mensual per cápita estimado por las encuestas periódicas de Caracterización Socioeconómica (CASEN), cada tres y, desde 2009, cada dos años- es inferior a la "línea de pobreza" o al ingreso mínimo establecido para satisfacer las necesidades básicas alimentarias y no alimentarias de una persona en ese 130 
mismo período; es decir, multiplicando el costo de alimentarse con base en la proporción aproximada del consumo de alimentos en el consumo total que arrojó la Encuesta de Consumo de los Hogares de 1978. En el caso de Chile, se ha utilizado, además, un ajuste para la población rural dado el supuesto de autoproducción de una parte de los alimentos en ese sector (el coeficiente para zonas urbanas es de 2.0, calculado con base en la Encuesta de Presupuestos Familiares de 1987, mientras para las zonas rurales se fijó en 1,75, siguiendo el criterio de la CEPAL).

El Gobierno de Chile solicitó desde 1987 a la CEPAL, además, que realice un ajuste de las cifras de ingresos de las familias a partir de información sobre el ingreso nacional que entrega el Banco Central, dando lugar a una estimación conjunta de la pobreza hasta 2010. Suele mencionarse que entre 1990 y 2013 la pobreza absoluta en Chile bajó de 38,6\% a 7,8\% de la población, mientras la indigencia o extrema pobreza lo hizo de $13,0 \%$ a 2,5\%, utilizando la metodología de líneas de pobreza de la CEPAL. Esta disminución reflejó el crecimiento de los ingresos que experimentó la economía chilena en el mismo período (el PIB se multiplicó por 3,2 veces, mientras el PIB por habitante pasó de 9029 dólares de 2011 en 1990 a 21783 dólares de 2011 en 2013, de acuerdo con las cifras del Wolrd Economic Outlook del Fondo Monetario Internacional de abril de 2018).

La publicación de los datos para 2009 y 2011 en Chile se produjo en medio de una discrepancia metodológica entre la CEPAL y el Gobierno. Aunque la información de base utilizada fue la misma (la encuesta de consumo de hogares de 1987-88 y la encuesta de ingresos CASEN de 2009), la CEPAL concluyó que la pobreza cayó en 2009 respecto de 2006, mientras para el Gobierno (conformado por una nueva coalición entre 2010 y 2014), en cambio, la pobreza subió. Esta es una ilustración de cuan dependiente de las opciones de medición estadística resulta ser la evaluación de los cambios en la pobreza. La práctica tradicionalmente utilizada por la CEPAL (2011) para computar la pobreza y la indigencia en cada uno de los años para los que se dispone de una medición de los ingresos es la actualización del valor de las líneas originales para reflejar la variación de los precios de los bienes y servicios, considerando el cambio experimentado por los precios de los alimentos.

Con esta práctica, la relación entre las líneas de pobreza y de indigencia permanecía constante. No obstante, a partir de 2007, y para todos los países, la línea de indigencia es actualizada por la CEPAL mediante la variación del componente alimentos del Índice de Precios al Consumidor (IPC), mientras que la parte de la línea de pobreza que corresponde al gasto en bienes no alimentarios se actualiza mediante la variación del IPC correspondiente. Desde 2007 en adelante, la diferencia entre las líneas de indigencia y de pobreza ya no es constante. Este cambio en el criterio de actualización del valor monetario de las líneas de pobreza se originó en el fuerte incremento de los precios de alimentos registrado a nivel regional en 2007 y 2008 . En el caso de Chile, mientras en el trienio 2006-2009 el IPC de los alimentos creció en 32,4\%, el del resto de los bienes solo lo hizo en $6,4 \%$. 
Tabla 1.

Chile, índices de pobreza (porcentaje de la población) a partir de encuesta de ingresos familiares CASEN

\begin{tabular}{|c|c|c|c|c|c|c|c|}
\hline Índices & 1990 & 1996 & 2006 & 2009 & 2011 & 2013 & 2015 \\
\hline CEPAL, Índice de Pobreza & 38,6 & 23,2 & 13,7 & 11,4 & 10,9 & 7,8 & - \\
\hline CEPAL, Índice de Indigencia & 13,0 & 5,7 & 3,2 & 3,6 & 3,1 & 2,5 & - \\
\hline MIDEPLAN-MDS, Índice de Pobreza Tradicional & 38,6 & 23,2 & 13,7 & 15,1 & 14,4 & - & - \\
\hline MIDEPLAN-MDS, Índice de Extrema Pobreza Tradicional & 13,0 & 5,7 & 3,2 & 3,7 & 2,8 & - & - \\
\hline Ministerio de Desarrollo Social, Índice de Pobreza 2013 & - & - & 29,1 & 25,3 & 22,2 & 14,4 & 11,7 \\
\hline $\begin{array}{l}\text { Ministerio de Desarrollo Social, Índice de Extrema } \\
\text { Pobreza } 2013\end{array}$ & - & - & 12,6 & 9,9 & 8,1 & 4,5 & 3,5 \\
\hline $\begin{array}{l}\text { Ministerio de Desarrollo Social, Índice de Pobreza } \\
\text { Muldimensional } 2013\end{array}$ & - & - & - & 27,5 & 24,3 & 20,4 & 19,1 \\
\hline $\begin{array}{l}\text { Ministerio de Desarrollo Social, Índice de Pobreza } \\
\text { Muldimensional } 2015\end{array}$ & - & - & - & - & - & - & 20,9 \\
\hline $\begin{array}{l}\text { OCDE, Pobreza relativa antes de impuestos y } \\
\text { transferencias ( } 50 \% \text { de la mediana) }\end{array}$ & - & - & 22,6 & 20,1 & 19,2 & 18,5 & 18,0 \\
\hline $\begin{array}{l}\text { OCDE, Pobreza relativa después de impuestos y } \\
\text { transferencias ( } 50 \% \text { de la mediana) }\end{array}$ & - & - & 19,2 & 17,8 & 18,4 & 16,8 & 16,1 \\
\hline $\begin{array}{l}\text { OCDE, Pobreza relativa antes de impuestos y } \\
\text { transferencias ( } 60 \% \text { de la mediana) }\end{array}$ & - & - & 29,2 & 26,3 & 25,7 & 24,6 & 23,9 \\
\hline $\begin{array}{l}\text { OCDE, Pobreza relativa después de impuestos y } \\
\text { transferencias ( } 60 \% \text { de la mediana) }\end{array}$ & & & 26,3 & 24,4 & 25,2 & 23,8 & 23,3 \\
\hline $\begin{array}{l}\text { Banco Mundial, Tasa de Pobreza menos de 5,5 US\$ por } \\
\text { día (PPP 2011) }\end{array}$ & 45,6 & 30,8 & 22,8 & 20,5 & 16,4 & 10,1 & 10,1 \\
\hline $\begin{array}{l}\text { Banco Mundial, Tasa de Pobreza menos de 3,2 US\$ por } \\
\text { día (PPP 2011) }\end{array}$ & 22,3 & 12,2 & 7,3 & 6,7 & 4,7 & 2,6 & 3,1 \\
\hline $\begin{array}{l}\text { Banco Mundial, Tasa de Pobreza, menos de 1,9 US\$ por } \\
\text { día (PPP 2011) }\end{array}$ & 7,6 & 3,7 & 2,4 & 2,6 & 1,6 & 0,9 & 1,3 \\
\hline
\end{tabular}

Fuentes: Bases de datos de CEPAL (2018), Ministerio de Desarrollo Social de Chile (2015), OCDE (2018) y Banco Mundial (2018), consultadas en enero de 2018, con cálculos realizados a partir de la Encuesta de Caracterización Socioeconómica (CASEN) del Gobierno de Chile. En cursivas los datos para extrema pobreza.

El Gobierno de Chile del período 2010-2014 no adoptó para la medición de 2009 el cambio introducido por la CEPAL y continuó actualizando el valor del conjunto de la línea de pobreza (alimentos y otros bienes y servicios) por la variación del precio de los alimentos, con lo que la tasa de pobreza resultante fue de $15,1 \%$ y no de $11,4 \%$ (ver Tabla 1). En cambio, la indigencia aumentó para el gobierno chileno de 3,2\% a 3,7\% entre 2006 y 2009 y a un 3,7\% para la CEPAL, pues ambas metodologías utilizaron los mismos ingresos medidos por la encuesta CASEN y un indicador similar de precios de los alimentos.

132 
La medición de 2011 suscitó nuevas controversias. La encuesta CASEN se aplicó en una fecha que permitió considerar la incidencia de un bono monetario entregado por el Gobierno en diciembre, sin promediarlo con el resto de los meses del año, con lo que se sobrestimó el ingreso de los más pobres. Además, se agregó nuevas fuentes de ingreso antes no consideradas. Se produjo en el caso de la encuesta de 2011 una divergencia entre el gobierno y la CEPAL mayor que la de 2009 en el cálculo de la indigencia (porcentaje de personas que no tienen ingresos suficientes para cubrir la compra de una canasta básica de alimentos).

Para el Ministerio de Desarrollo Social chileno la indigencia bajó de 3,7\% a 2,8\% entre 2009 y 2011, mientras para la CEPAL la disminución fue de $3,6 \%$ a $3,1 \%$ en el mismo período. Estos hechos produjeron una fuerte controversia pública sobre la credibilidad de los datos de pobreza, mientras la CEPAL decidió suspender temporalmente su colaboración técnica con el gobierno chileno de la época en esta materia.

El Gobierno chileno terminó por decidir suspender la encuesta prevista para 2012 y realizarla al año siguiente, una vez que se hubieran revisado los criterios metodológicos por una comisión de expertos. Así, la precipitación del Gobierno de 2010-2014 de Sebastián Piñera para procurar reflejar un aumento de la pobreza en el gobierno de 2006-2010 de Michelle Bachelet terminó, paradójicamente, por situar el nivel de pobreza oficial en 2011 en un nivel más alto que la medición de la CEPAL, con cifras, a la postre, menos favorables para la gestión del gobierno de Sebastián Piñera.

Otra ilustración de la variabilidad de la medición de pobreza, según distintas opciones metodológicas, es el hecho de que, siempre utilizando la misma encuesta CASEN, la medición del Banco Mundial arroja estimaciones sustancialmente inferiores en el caso de la "línea internacional de pobreza" a las mencionadas anteriormente (solo 1,3\% de la población en condiciones de pobreza en 2015, en el caso de Chile, en la línea de 1,9 dólares por habitante al día) y bastante disímiles entre sí, en el caso de las dos siguientes líneas (de 3,2 y 5,5 dólares por habitante al día). No obstante, esta última presenta órdenes de magnitud relativamente semejantes a los cálculos recientes de pobreza monetaria del gobierno de Chile. Así, el uso del enfoque de necesidades de alimentación y, a partir de ahí medir la capacidad de solventar alguna canasta de bienes para medir la magnitud de la pobreza, ha terminado, en el caso de Chile, envuelto en diversas controversias técnicas que han mermado la credibilidad de las cifras oficiales de pobreza ante la opinión pública.

La incorporación de Chile a la OCDE en 2011 aportó la medición de la pobreza relativa que realiza esta organización, utilizando las mismas encuestas de ingresos y estimando el porcentaje de la población en situación de pobreza con el umbral de los hogares con ingresos inferiores al 50\% y $60 \%$ de la mediana del ingreso disponible antes y después de impuestos y transferencias. Para la OCDE (según su Income Distribution Database), en 2009 la pobreza relativa afectaba un 18,4\% de 
la población después de impuestos y transferencias, considerando el $50 \%$ de la mediana de ingresos como línea de pobreza, y a $25,3 \%$, considerando al $60 \%$ de la mediana de ingresos. Esta cifra es considerablemente mayor que las que resultan de las diversas estimaciones de pobreza absoluta reseñadas, pero también conserva, como en el caso del Banco Mundial, el mismo signo de cambio entre 2006 y 2009 que la CEPAL. Desde 2009, la pobreza relativa según la OCDE, ha variado poco, de $26,3 \%$ a $23,3 \%$, entre 1996 y 2015 , en el caso de la línea de $60 \%$ de la mediana de ingresos disponibles, y de $19,2 \%$ a $16,1 \%$, en el caso de la línea de $50 \%$ de la mediana de ingresos disponibles, la más usada por la OCDE en sus informes sobre sus países miembros (ver nuevamente Tabla 1). La CEPAL (2012) también ha incorporado esta perspectiva y constata que solo en seis países de América Latina la pobreza relativa es superior a la absoluta, incluido Chile.

\section{Las nuevas mediciones gubernamentales de pobreza desde 2015}

El Ministerio de Desarrollo Social dio a conocer en 2015 los resultados de la encuesta de Caracterización Socio-Económica (CASEN) correspondiente a 2013 con cambios metodológicos sustanciales en la medición de la pobreza, nuevamente con la asesoría técnica de la CEPAL y también de la Iniciativa de Oxford para la Pobreza y el Desarrollo Humano (OPHI). Se tomaron en cuenta, por primera vez, escalas de equivalencia en el consumo del hogar, considerando que los niveles de consumo de los jefes de hogar suele ser superior al de niños y personas de edad. El valor de la elasticidad de equivalencia utilizada por el Ministerio de Desarrollo Social para tomar en cuenta la presencia de economías de escala en el consumo es de 0,7.

Se considera desde 2015 que un hogar está en situación de pobreza si su ingreso mensual por persona es inferior a la "línea de pobreza por persona equivalente" o ingreso mínimo establecido para satisfacer las necesidades básicas alimentarias y no alimentarias de una persona equivalente en ese mismo período. El valor de la línea de pobreza fue estimado sobre la base del gasto de los hogares en el estrato de referencia determinado en la Encuesta de Presupuestos Familiares 2011-2012, excluyendo los gastos en alcohol y tabaco y el gasto en bienes y servicios adquiridos por menos del $10 \%$ de los hogares.

La nueva canasta de alimentos utilizada considera un umbral de requerimientos de 2.000 calorías diarias promedio por persona, mientras la canasta tradicional consideraba requerimientos calóricos básicos para zonas urbanas de $2.176 \mathrm{Kcal}$ por persona al día, y para zonas rurales, de 2 $236 \mathrm{Kcal}$, con un promedio ponderado de $2.187 \mathrm{Kcal}$, canasta basada en los patrones de consumo observados para un grupo de referencia de la población urbana. La nueva composición de la canasta fue determinada a partir del gasto de aquel grupo de la población correspondiente al quintil de hogares de menores ingresos. Este grupo de referencia gastaba en 2012 un 37,3\% de su presupuesto en alimentos.

El valor de la línea de pobreza extrema equivale en la nueva metodología a dos tercios de la línea de pobreza, lo que es cercano al gasto que realizan los hogares del grupo de referencia en 134 
alimentación, vivienda y vestuario. Por otra parte, se calculan los ingresos provenientes del trabajo y de la propiedad de activos (incluyendo una imputación de arriendo a los propietarios de vivienda), las prestaciones de los seguros sociales y la asistencia social y las diversas transferencias corrientes entre hogares. Se imputan los datos faltantes en las variables de ingreso de trabajadores dependientes e independientes, así como también para jubilaciones y rentas vitalicias, aunque ahora sin ajustes con la cuenta institucional de hogares de las cuentas nacionales. Se mantiene una imputación por arriendo para las personas con casa propia, aunque esta imputación es metodológicamente impugnada por autores como Balcázar, Ceriani, Olivieri, \& Ranzani (2017) arguyendo que las mediciones de ingresos no deben incluir estimaciones de stock de activos y su conversión en flujos monetarios.

Adicionalmente, se incorporó desde 2015 una medición de pobreza multidimensional. El Gobierno de Chile incluyó en la medida de pobreza multidimensional en una primera etapa (encuesta 2013) cuatro dimensiones (educación, salud, vivienda y trabajo) y más tarde (encuesta 2015) cinco dimensiones (se agregó la dimensión de redes y cohesión social), lo que hace variar sustancialmente las mediciones tradicionales de pobreza. La primera dimensión es la educación. Se considera que un hogar es carente en escolaridad si al menos uno de sus integrantes mayores de 18 años ha alcanzado menos años de escolaridad que los establecidos por la ley. Se considera que un hogar es carente por asistencia escolar si al menos uno de sus integrantes de 4 a 18 años no está asistiendo a un establecimiento educacional y no ha egresado de cuarto medio o si al menos un integrante de 6 a 26 años no asiste a un establecimiento educacional. Se considera que un hogar es carente por rezago escolar si al menos uno de sus integrantes de 21 años o menos asiste a educación básica o media en alguna de sus dos modalidades y se encuentra retrasado dos años o más con respecto al curso que le corresponde de acuerdo a su edad.

En la dimensión de salud se considera que un hogar es carente en nutrición en niños si al menos uno de sus integrantes de 0 a 6 años posee sobrepeso u obesidad o está en desnutrición o riesgo de desnutrición. Se considera que un hogar es carente en adscripción a un sistema de salud si al menos uno de sus integrantes no está afiliado a un sistema previsional de salud y no tiene otro seguro de salud. Se considera que un hogar es carente en acceso a atención de salud si al menos uno de sus integrantes tuvo un problema de salud en los últimos tres meses y no tuvo consulta ni atención por falta de tiempo, dinero o dificultad para obtener la atención.

En la dimensión de vivienda se considera que un hogar es carente por hacinamiento si el número de personas en el hogar por dormitorio de uso exclusivo es mayor o igual a 2,5. Se considera que un hogar es carente si la vivienda presenta muros, techos o suelos en mal estado o no tiene acceso a agua potable procedente de red pública, en el caso de áreas urbanas, o a agua procedente de red o sistema de distribución proveniente de fuentes subterráneas o superficiales, en áreas rurales, y si no tiene un servicio de eliminación de excretas adecuado. 
En la dimensión de trabajo y seguridad social se considera que un hogar es carente en ocupación si al menos uno de sus integrantes mayores de 18 (o menor de 19 si ha completado la enseñanza media) está desocupado. Se considera que un hogar es carente en seguridad social si al menos uno de sus integrantes de 15 años o más que se encuentra ocupado no cotiza en el sistema previsional y no es trabajador independiente con educación superior completa. Se considera que un hogar es carente en jubilaciones si al menos uno de sus integrantes no percibe una pensión contributiva (mujeres de 60 años o más y hombres de 65 años o más), o no contributiva (pensión básica solidaria para personas de 65 años o más o pensiones de reparación) y no recibe otros ingresos.

El Ministerio de Desarrollo Social (2015) definió una ponderación equivalente para cada dimensión y otro tanto para los indicadores al interior de cada una. Se considera que un hogar está en situación de pobreza multidimensional si tiene al menos $25 \%$ de carencias. Un $20,4 \%$ de la población se encontraba en 2013 en estado de pobreza multidimensional, a comparar con un $14,4 \%$ en estado de pobreza según ingresos, con la nueva metodología, y con un $7,8 \%$ con la metodología tradicional de la CEPAL. En la encuesta de 2015, el Gobierno amplió la dimensión de vivienda incorporando elementos del entorno y agregó una quinta dimensión, la de redes y cohesión social. Las ponderaciones de las dimensiones tradicionales se establecieron en 22,5\%, mientras la dimensión redes y cohesión social se ponderó en un $10 \%$ en el índice compuesto, sin que se dieran a conocer mayores explicaciones en la materia. Dentro de cada dimensión, los indicadores tienen igual peso: 7,5\%, en el caso de las dimensiones tradicionales, y 3,33\% en el de redes y cohesión social. Los hogares que acumulan un 22,5\% o más de carencias se encuentran en situación de pobreza multidimensional. Este nuevo índice incrementó de 19,1\% a 20,9\% la población en estado de pobreza multidimensional (ver nuevamente la Tabla 1).

\section{Conclusiones}

El análisis de las diversas metodologías de medición de la pobreza en Chile y sus cambios recientes pone en evidencia que, si bien están aplicadas a la misma encuesta de los ingresos de las familias realizada por el Gobierno periódicamente, los rangos de diferencia en los resultados son elevados y dependientes de las diferentes definiciones de pobreza absoluta, relativa y multidimensional utilizadas. Estos resultados confirman la proposición general de Sen (1992) sobre la alta variabilidad de los resultados de las mediciones de pobreza según las definiciones canónicas utilizadas, lo que debe ser subsanado con la recomendación de que "casi no queda más que aceptar el elemento de arbitrariedad presente en la descripción de la pobreza y hacerlo tan transparente como sea posible" (p. 6).

136 


\section{Referencias}

Alkire, S. \& Foster, J. (2007). Recuento y medición multidimensional de la pobreza. En OPHI Working Paper 7, Universidad de Oxford. Recuperado de http://www.ophi.org.uk/wpcontent/uploads/ophi-wp7-es.pdf

Alkire, S. \& Foster, J. (2011). Understandings and misunderstandings of multidimensional poverty measurement. En OPHI Working Paper 43, Universidad de Oxford. Doi: https://doi.org/10.1007/s10888-011-9181-4

Arrow, K. (2015). Which inequalities matter and which taxes are appropriate? En Out of the Crooked Timber of humanity, no straight thing was ever made. Recuperado de http://crookedtimber.org/2015/12/17/which-inequalities-matter-and-which-taxes-areappropriate/

Balcázar, C., F., Ceriani, L., Olivieri, S., \& Ranzani, M. (2017). Rent-imputation for welfare measurement: A review of methodologies and empirical findings. The Review of Income and Wealth, 4(63), 881-898. Doi: https://doi.org/10.1111/roiw.12312

Banco Mundial. (2018). Datos Banco Mundial. Recuperado de https://datos.bancomundial.org/indicador/SI.POV.DDAY?locations=1W\&start=1981\&e $\underline{\text { nd}}=2013 \&$ view $=$ chart.

Comisión Económica para América Latina y el Caribe. (2011). Comunicado. Recuperado de https://www.cepal.org/es/comunicados/la-cepal-describe-metodologia-paramedicion-de-la-pobreza-en-chile-en-2009.

Comisión Económica para América Latina y el Caribe. (2012). Panorama social de América Latina. Recuperado de http://www.cepal.org/publicaciones/xml/5/48455/PanoramaSocial2012Docl-Rev.pdf

Comisión Económica para América Latina y el Caribe. (2018). CEPALSTAT. Recuperado de http://estadisticas.cepal.org/cepalstat/WEB_CEPALSTAT/estadisticasIndicadores.asp?i dioma $=$ e.

Meyer, B. D., \& Sullivan, J.X. (2012). Identifying the disadvantaged: Official poverty, consumption poverty, and the new supplemental poverty measure. Journal of Economic Perspectives, 3(26), 111-136. Doi: https://doi.org/10.1257/jep.26.3.111 
Ministerio de Desarrollo Social de Chile. (2015). Nueva metodología de medición de la pobreza por Ingresos y multidimensional. Serie Documentos Metodológicos28, 1-58. Recuperado de http://observatorio.ministeriodesarrollosocial.gob.cl/documentos/Nueva_Metodologi a de Medicion de Pobreza.pdf

Organisation for Economic Co-operation and Development. (2018). Income Distribution Database. Recuperado de http://www.oecd.org/social/income-distributiondatabase.htm

Ravallion, M. (2011). On multidimensional indices of poverty. En Policy Research working paper WPS 5580. Washington, D.C.: Banco Mundial. Recuperado de http://documents.worldbank.org/curated/en/458211468336027757/Onmultidimensional-indices-of-poverty

Ravallion, M., \& Chen, S. (2011). Weakly relative poverty. The Review of Economics and Statistics, 4(93), 1251-1261. Doi: https://doi.org/10.1162/REST a 00127

Sen, A. K. (1992). Sobre conceptos y medidas de pobreza. Comercio Exterior, 42(4). Recuperado de $\quad$ http://www.derechoshumanos.unlp.edu.ar/assets/files/documentos/sobreconceptos-y-medidas-de-pobreza.pdf. 